\title{
Short-term Side Effects of Pulse Steroid Treatment in Children
}

\author{
Gulbaran Koncak, Orkun Tolunay, Asena Unal, Can Celiloglu and Umit Celik \\ Department of Pediatrics, University of Health Sciences, Adana City Training and Research Hospital, Adana, Turkey
}

\begin{abstract}
This study is aimed to evaluate pediatric patients, who were hospitalised in the Department of Pediatrics, University of Health Sciences, Adana City Training and Research Hospital, Turkey, between January, 2019 and January, 2020, and treated with pulse steroid therapy and the early side effects of their treatment. The fasting blood qlucose levels of the patients during treatment were statistically significantly higher than those prior to the treatment. The most common side effects observed in the patients were dermatological (48.5\%), psychiatric (31.4\%), and gastrointestinal (31.4\%). Hypertension was detected in seven patients (20\%) after treatment; and continued in three, who subsequently underwent antihypertensive treatment. Pulse steroid treatment was administered for a median of five days (3-11 days). It was found that 24 patients responded to treatment, 11 patients did not respond, and one patient died. There is a shortage of studies in literature on pulse steroid therapy and its side effects, especially focusing on children. Multicentre and randomised controlled studies are needed comprising different patient groups to evaluate the efficacy and complications associated with its use.
\end{abstract}

Key Words: Children, Side effect, Pulse steroid treatment.

How to cite this article: Koncak G, Tolunay O, Unal A, Celiloglu C, Celik U. Short-term Side Effects of Pulse Steroid Treatment in Children. J Coll Physicians Surg Pak 2022; 32(02):262-264.

Corticosteroids have been used successfully for more than half a century to treat various inflammatory, malignant and autoimmune diseases. ${ }^{1}$ The aim of pulse steroid treatment is to increase and accelerate the therapeutic effect, and to reduce side effects. Pulse steroid therapy is now being applied in many situations in which inflammation and autoimmunity are seen, such as for the treatment of nephrological, hematological, rheumatological, neurological and allergic diseases. Today, pulse steroid therapy has found a place in the treatment of many hematological and autoimmune diseases, where more successful results are being obtained than with standard-dose steroid therapy. ${ }^{1}$ Although steroid use is now quite common, there are some undesired side effects.

The aim of the study was to evaluate pediatric patients, who were treated with pulse steroid therapy and determine the early side effects of the treatment.

This retrospective study included patients aged one month to 18 years, who were hospitalised in the Department of Pediatrics, University of Health Sciences, Adana City Training and Research Hospital, Turkey and administered pulse steroid therapy between January, 2019 and January, 2020.

Correspondence to: Dr. Asena Unal, Department of Pediatrics, University of Health Sciences, Adana City Training and Research Hospital, Adana, Turkey

E-mail: asenaunal@yahoo.com

Received: September 15, 2021; Revised: December 07, 2021;

Accepted: December 10, 2021

DOI: https://doi.org/10.29271/jcpsp.2022.02.262
Patients whose data could not be reached, were excluded from the study. The study was granted ethical approval by the University of Health Sciences, Adana City Training and Research Hospital Clinical Trials Ethics Committee. The statistical analysis of the study data was performed using IBM SPSS Statistics (Version 20.0. Armonk, NY: IBM Corp.). The normal distribution of the numerical measurements in the study group was examined withthe Kolmogorov-Smirnov test. Descriptivestatistics related to numerical parametric variables were presented as mean \pm standard deviation. Non-parametric variables were presented as median (interquartile range) and categorical variables were expressed as counts and percentage (\%). Pairedsamples T-test was used in the dependent groups and Wilcoxon Signed-Rank test was used for non-parametric variables. The limit of significance was accepted as $p<0.05$.

The study included 35 patients, of which $19(54.3 \%)$ were males, 17 (45.7\%) were females; and the median age was 8 (IQR 5-11) years. The most common hospitalisation diagnoses of the patients were encephalitis (22.8\%), juvenile idiopathic arthritis (JIA), and immune thrombocytopenia (ITP) in $14.2 \%$. While, polyarteritis nodosa, dermatomyositis, hemolytic anemia and purpura diagnosis were found in two patients (5.7\%).

When the patients were grouped according to their diagnoses, 15 patients $(42.9 \%)$ were treated for neurological, 12 patients $(34.3 \%)$ for rheumatological, seven patients $(20 \%)$ for hematological, and one patient (2.9\%) for gastrointestinal system diseases. All patients in the study were given methylprednisolone. 
The median fasting blood glucose levels of the patients, who underwent pulse steroid treatment, were 87 (83-104) mg/dl before the treatment, $123(110-150) \mathrm{mg} / \mathrm{dl}$ during the treatment, and 93 (88-103) mg/dl after the treatment. While there was no statistically significant difference between the pre-treatment and post-treatment measurements $(p=0.195)$, the difference between the fasting blood glucose measurements before and during treatment was statistically significantly higher $(p<0.001)$. When the post-treatment white blood cell, neutrophil, lymphocyte and monocyte values of the patients undergoing pulse steroid treatment were compared with the pre-treatment values, the difference between groups was found to be statistically significantly higher $(p=0.007,0.038$, $0.033,0.003)$. There was no statistically significant difference between the platelet, sodium, potassium, calcium, phosphorus and uric acid levels of the patients before and after treatment (Tablel).

Table l: Laboratory findings of patients treated with pulse steroids.

\begin{tabular}{|c|c|c|c|}
\hline & Pre-treatment & Post-treatment & $\mathbf{P}$ \\
\hline White blood cell $\left(x 10^{\wedge} 3 / \mu \mathrm{L}\right)^{*}$ & $8.9(6.7-14.9)$ & $13.8(8.2-16.6)$ & $0.007^{* *+1}$ \\
\hline Neutrophil $\left(x 10^{\wedge} 3 / \mu \mathrm{L}\right)^{*}$ & $6.1(3.9-11.2)$ & $9.3(4.8-13.3)$ & $0.038^{* * *+}$ \\
\hline Lymphocyte $\left(x 10^{\wedge} 3 / \mu \mathrm{L}\right){ }^{*}$ & $2.3(1.1-3.2)$ & $2.5(1.5-4.4)$ & $0.033^{k * *+1}$ \\
\hline Monocyte $\left(x 10^{\wedge} 3 / \mu \mathrm{L}\right)^{*}$ & $0.7(0.4-0.9)$ & $0.9(0.6-1.3)$ & $0.003^{k+*+1}$ \\
\hline Platelet $\left(\times 10^{\wedge} 3 / \mu \mathrm{L}\right){ }^{*}$ & $310(213-391)$ & $303(233-456)$ & $0.427^{* *+1}$ \\
\hline Sodium $(\mathrm{mm} / \mathrm{l})^{* *}$ & $137(134-138.25)$ & $136(134-139)$ & $0.473^{k * *+1}$ \\
\hline Potassium $(\mathrm{mm} / \mathrm{l})^{* *}$ & $4.15(3.7-4.52)$ & $4.4(4.1-4.7)$ & $0.072^{k *+1}$ \\
\hline Calcium (mg/dl) ${ }^{* *}$ & $9.4(8.8-9.82)$ & $9.15(8.8-9.52)$ & $0.180^{* *+1}$ \\
\hline Phosphorus (mg/dl) ${ }^{* *}$ & $4.2 \pm 1.27$ & $4.1 \pm 1.09$ & $0.792^{* *+\infty}$ \\
\hline Uric acid $(\mathrm{mg} / \mathrm{dl})^{*}$ & $2.9(2-4.3)$ & $3.3(2.3-4.2)$ & $0.459^{k+1+1}$ \\
\hline
\end{tabular}

Dermatological findings were observed in 17 patients $(48.5 \%)$, edema in 16 patients (45.7\%), erythema in 12 patients (34\%), hirsutism in eight patients $(22.8 \%)$, acne in four patients $(11.4 \%)$, striae in four patients $(11.4 \%)$, and acanthosis in one patient (2.8\%). Some patients had more than one dermatological findings. Psychiatric findings were observed in 11 patients (31.4\%), mood disorders in nine patients $(25.7 \%)$, and anxiety disorders in two patients (5.7\%). Gastrointestinal symptoms were observed in 11 patients (31.4\%). No Gl bleeding was detected. Hypertension was detected in seven patients $(20 \%)$ after treatment, and continued in three (8.5\%), who subsequently underwent antihypertensive treatment (continuing for an average of six months). Sinus tachycardia was observed in one patient during steroid infusion, but no treatment was required.

Pulse steroids were administered to the patients over 10 $\mathrm{mg} / \mathrm{kg} /$ day methylprednisolone, but varied according to the disease group and clinic. The pulse steroid therapy was continued for a minimum of 3 days and a maximum of 11 days, with a median duration of five days. In summary, 12 patients $(34.3 \%)$ received treatment for $1-3$ days, 13 patients (37.1\%) for 4-6 days, and 10 patients (28.6\%) for 7-11 days. A comparison of the duration of treatment revealed no statistically significant difference in the patients with psychiatric, dermatological or GIS symptoms; but a statistically significant difference was observed in those with cardiac (hypertension) symptoms $(p=0.676,0.410,0.177,<0.001)$.

When the response to pulse steroid treatment was evaluated, 24 patients $(68.6 \%)$ were found to respond to treatment and 11 patients $(31.4 \%)$ did not.

Corticosteroids can cause cognitive disorders, behavioural changes, and psychiatric diseases. In a study conducted by Hall et al., neuropsychiatric symptoms were observed in more than $50 \%$ of the patients undergoing steroid treatment; while in similar studies, severe side effects were observed in approximately $6 \%$, and mild/moderate side effects in approximately $28 \%$ of the patients. ${ }^{2}$ In the present study, neuropsychiatric symptoms were observed in $31.4 \%$ of the patients, although most complaints were of mild symptoms. When we compared the neuropsychiatric symptoms seen in the patients in the present study with the duration/dose of pulse steroid use, no significant relationship could be identified.

The dermatological side effects, associated with steroid treatment, vary according to the duration of treatment, treatment dose, and individual characteristics. ${ }^{3}$ In an observational study of glucocorticoids involving patients with rheumatoid arthritis (RA), parchment-like appearance of the skin and ecchymosis side effects were reported in $10 \%$ and $17 \%$ of patients, respectively. ${ }^{3}$ In the present study, skin changes were observed in 16 patients (45.7\%).

Studies have found the frequency of GI complaints to increase two- to three-folds with steroid treatment. ${ }^{4}$ Although GIS complaints are increased among patients receiving steroids, it has been shown that less than $2 \%$ of these patients develop peptic ulcers, and GI bleeding is very rare. ${ }^{4}$ In the present study, all patients used proton pump inhibitors; and gastrointestinal complaints were observed in $31.4 \%$ of the patients.

Hypertension is a well-known side effect of glucocorticoids, and occurs in approximately $20 \%$ of patients exposed to exogenous glucocorticoids. ${ }^{5}$ In a study conducted by Huscher, steroid treatment was shown to increase the risk of hypertension. ${ }^{3}$ In the present study, seven patients (20\%) were found to be hypertensive, when assessed following treatment.

Metabolic-endocrine system side effects can be seen with the use of steroids. In a study conducted by Olefsky, it is revealed that the acute effects on glucose tolerance were much more pronounced than the chronic effects, and that no diabetes developed in a patient whose blood glucose was normal before glucocorticoid use. ${ }^{6}$ Similarly, in a prospective study, an average increase in fasting blood glucose of $30-54 \%$ was noted after the first dose of treatment, with a slow spontaneous return to baseline values in nondiabetic patients upon subsequent doses. ${ }^{2}$ In our study, although the blood glucose levels, measured during the treatment, were statistically significantly higher than before the treatment, no hyperglycemia requiring treatment developed in any of the patients.

It can be predicted that pulse steroid therapy, which has been in use since the 1940s, will increase in popularity among physi- 
cians in the future. In pediatric disease groups, in particular, the fact that treatment doses and durations are not fully standardised, makes it difficult to monitor treatment-related side effects. Accordingly, guidelines should be established for the treatment of side effects in patients. Prospective, multicenter studies are needed involving different pediatric disease groups to monitor the effects of pulse steroid therapy.

\section{ACKNOWLEDGMENT:}

This work was done at University of Health Sciences, Adana City Training and Research Hospital and has not been presented elsewhere.

\section{ETHICALAPPROVAL:}

The study was granted ethical approval by the University of Health Sciences, Adana City Training and Research Hospital ClinicalTrials Ethics Committee.

\section{CONFLICT OF INTEREST:}

The authors declared no conflict of interest.

\section{AUTHORS' CONTRIBUTION:}

GK, OT, AU, CC, UC: Conception, design, acquisition, analysis, interpretation of data, drafting, revising it critically for important intellectual content, and final approval of the version to be published.
All the authors agreed to be accountable for all aspects of the work in ensuring that questions related to the accuracy or integrity of any part of the work are appropriately investigated and resolved.

\section{REFERENCES}

1. Sinha, A, Bagga A. Pulse steroid therapy. Indian J Pediatr 2008; 75:1057-66.

2. Chibane S, Feldman-Billard S, Rossignol I, Kassaei R, Mihoubi-Mantout F, Heron E. Short-term tolerance of three days pulse methyprednisolone therapy: A prospective study in 146 patients. Rev Med Interne 2005; 26(1):20-6. doi: 10.1016/j.revmed.2004.09.025.

3. Huscher D, Thiele K, Gromnica-Ihle E, Hein G, Demary W, Dreher $\mathrm{R}$, et al. Dose-related patterns of glucocorticoidinduced side effects. Ann Rheum Dis 2009; 68(7): 1119-24. doi: 10.1136/ard.2008.092163.

4. Hernandez-Diaz S, Rodriguez LA. Steroids and risk of upper gastrointestinal complications. Am J Epidemiol 2001; 153(11):1089-93.

5. Whitworth JA. Mechanisms of glucocorticoid-induced hypertension. Kidney Int 1987; 31(5):1213-24. doi: 10.1038/ki.1987.131.

6. Olefsky JM, Kimmerling G. Effects of glucocorticoids on carbohydrate metabolism. Am J Med Sci 1976; 271:202-10. 